\title{
Анализ пороговых условий и эффективности генерации замкнутых мод в больших прямоугольных резонаторах на основе лазерных гетероструктур AIGaAs/GaAs/InGaAs
}

\author{
(c) А.А. Подоскин, Д.Н. Романович, И.С. Шашкин, П.С. Гаврина, З.Н. Соколова, \\ С.О. Слипченко, Н.А. Пихтин
}

Физико-технический институт им. А.Ф. Иофре Российской академии наук, 194021 Санкт-Петербург, Россия

E-mail: Podoskin@mail.ioffe.ru

Поступила в Редакцию 13 января 2021 г.

В окончательной редакции 18 января 2021 г.

Принята к публикации 18 января 2021 г.

\begin{abstract}
Представлена оценка пороговых условий и эффективности работы полупроводникового лазерного излучателя с большим прямоугольным резонатором $(1 \times 1$ мм) на основе гетероструктуры $\mathrm{AlGaAs} / \mathrm{GaAs} / \mathrm{InGaAs}$ для мощных полосковых лазеров, работающего на высокодобротных замкнутых модовых структурах. Предложены два варианта конструкции излучателей с различными характеристиками областей распространения лазерного излучения и показана возможность достижения дифференциальной эффективности, характерной для мощных полосковых лазеров, > 70\%.
\end{abstract}

Ключевые слова: замкнутая мода, лазерная гетероструктура, AlGaAs/GaAs/InGaAs.

DOI: 10.21883/FTP.2021.05.50838.9601

\section{1. Введение}

В прямоугольных кристаллах мощных лазеров полосковой конструкции на базе гетероструктур $\mathrm{AlGaAs} / \mathrm{GaAs} / \mathrm{InGaAs}$ известно явление возникновения замкнутых модовых структур (замкнутая мода 3M, internally circulating mode - ICM) за счет полного внутреннего отражения от всех четырех боковых граней кристалла [1,2]. Аналогичные модовые структуры многократно и подробно рассматривались для случаев прямоугольных микрорезонаторов с характерными размерами в единицы-десятки длин волн. В рамках данной работы рассматриваются большие прямоугольные кристаллы-резонаторы с характерными размерами в сотни длин волн и более. Возникновение генерации 3М в полосковых лазерах, конструктивно являющихся большими кристаллами прямоугольной формы, приводит к снижению эффективности генерации мод резонатора Фабри-Перо (ФПМ) и традиционно рассматривается исключительно как негативный для мощного полоскового лазера эффект. Однако результаты предыдущих исследований показали, что структуры 3М в больших прямоугольных резонаторах (до $1 \times 1$ мм и более) представляют собой гребенчатый спектр генерации с бо́льшими, нежели в случае ФПМ, межмодовыми расстояниями [3], что делает ЗМ потенциально привлекательным источником излучения для прикладных задач, где востребованы спектры в виде серии эквидистантных лазерных линий. Показанные в [3] изменения положения линий генерации ЗМ и межмодового расстояния при изменении геометрической конфигурации открывают перспективы использования кристаллов с управляемой геометрией $3 \mathrm{M}$ в качестве перестраиваемых источников излучения в ближнем инфракрасном диапазоне. Высокая степень подавления генерации ФПМ в полосковых лазерах при возникновении 3М говорит о ее высокой излучательной эффективности. При этом ЗМ характеризуется чрезвычайно низкой эффективностью вывода излучения из кристалла. Основными путями вывода излучения являются различные внутрирезонаторные дефекты и неоднородности, приводящие к рассеянию излучения, а также дефекты боковых сколов кристалла, нарушающие полное внутреннее отражение. Для обеспечения возможности эффективного использования излучения 3М в кристалл необходимо ввести элементы, обеспечивающие вывод излучения. В качестве таких элементов могут быть использованы одномерные или двумерные области с изменяющимся эффективным показателем преломления 3М, созданные в поперечном волноводе лазерной гетероструктуры. Создание таких областей может быть обеспечено за счет травления, многостадийной эпитаксии, а также их сочетания. Цель настоящей работы заключается в анализе пороговых условий и излучательной эффективности источника на основе кристалла с выводом излучения замкнутой моды.

\section{2. Лазерная гетероструктура и образцы}

В рамках данной работы рассматриваются пороговые условия и эффективность генерации ЗМ на примере лазерного кристалла (рис. 1), изготовленного на основе типичной гетероструктуры $\mathrm{AlGaAs} / \mathrm{GaAs} / \mathrm{InGaAs}$ на длину волны 1.06 мкм. Гетероструктура содержала нелегированный волновод $\mathrm{Al}_{0.1} \mathrm{Ga}_{0.9} \mathrm{As}$ толщиной 1.7 мкм, 


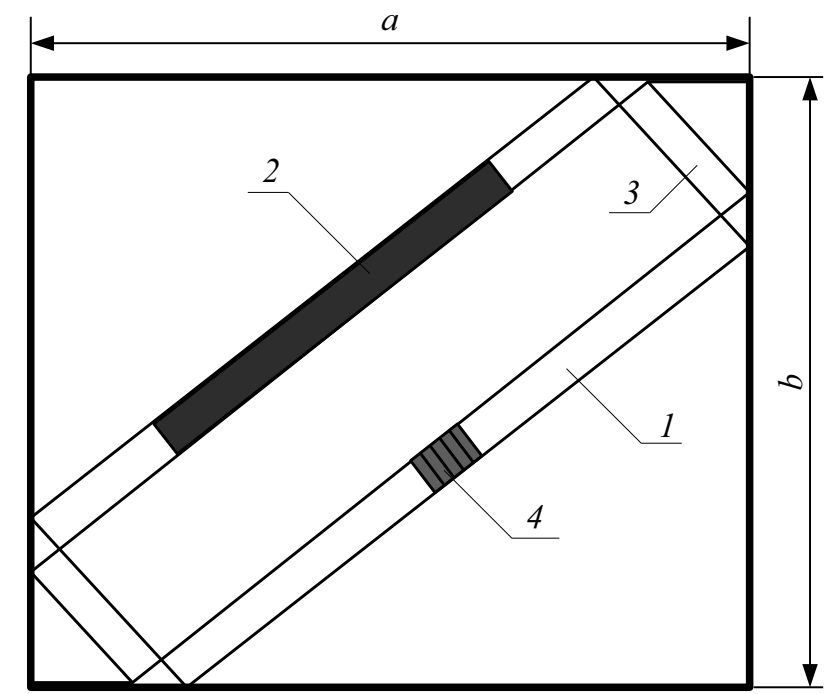

Рис. 1. Схематичное изображение модельного лазерного кристалла (вид сверху): 1 - область распространения замкнутой моды, 2 - область усиления, 3 - пассивная часть кристалла в области распространения 3М, 4 - область вывода излучения.

легированные $p$ - и $n$-эмиттеры $\mathrm{Al}_{0.25} \mathrm{Ga}_{0.75} \mathrm{As}$ толщинами 1.5 и 2 мкм соответственно. Активная область состояла из двух квантовых ям InGaAs толщиной 9 нм на длину волны 1065 нм. Для базовой характеризации гетероструктуры изготавливались лазеры со сплошным (для генерации 3М) и полосковым (для генерации ФПМ) контактами. В результате получены характеристическая плотность порогового тока $90 \mathrm{~A} / \mathrm{cm}^{2}$, внутренние оптические потери $0.7 \mathrm{~cm}^{-1}$ при внутреннем квантовом выходе > 95\%. Рассматриваемый модельный кристалл для генерации ЗМ содержит в себе три типа областей: область усиления, пассивную часть кристалла и область вывода излучения (рис. 1). Область усиления прокачивается током через стандартный омический контакт и характеризуется спектром усиления активной области и соответствующими внутренними оптическими потерями. Пассивная часть кристалла имеет соответствующий спектр поглощения, а область вывода характеризуется величиной потерь на вывод излучения $\left(\alpha_{\mathrm{CM}}^{\text {out }}\right)$. Область вывода излучения ЗМ можно рассмотреть как оптический делитель, отделяющий часть лазерного пучка и выводящий его из кристалла. Если принять за $\delta_{\text {out }}$ долю выводимого (отделяемого наружу из кристалла) излучения, то эквивалентный коэффициент оптических потерь на вывод излучения $\alpha_{\mathrm{CM}}^{\text {out }}$, вносимых элементом вывода в лазерную моду, можно записать, использовав закон Бугера, в виде: $\alpha_{\mathrm{CM}}^{\text {out }}=\left(1 / L_{\mathrm{CM}}\right) \ln \left(\delta_{\text {out }}\right)$, где $L_{\mathrm{CM}}-$ длина траектории $3 \mathrm{M}$ в рассматриваемом кристалле. Для случая 3М с однократным отражением от каждой грани (рис. 1) $L_{\mathrm{CM}}=\sqrt{a^{2}+b^{2}}$, где $a$ и $b-$ длины сторон кристалла (характерные размеры кристалла $1 \times 1$ мм в данной работе).

\section{3. Пороговые условия замкнутой моды}

Рассмотрим условия распространения 3М в различных частях модельного лазерного кристалла. На рис. 2 представлены характерные спектры генерации ФПМ и ЗМ для рассматриваемой лазерной гетероструктуры. Спектр 3М измерялся на кристаллах со сплошным омическим контактом размерами $500 \times 500$ мкм, спектр ФПМ - для полоскового лазера с длиной резонатора 2.5 мм и шириной апертуры 100 мкм. Наблюдаемый сдвиг линии ЗМ в длинноволновую сторону относительно ФПМ лежит в пределах типичных значений $5-20$ нм $[2,4]$. Спектральные зависимости усиления от концентрации для активных областей на основе квантовых ям хорошо описаны как экспериментально, так и теоретически [1,5-7]. Для оценки величин усиления и межзонного поглощения 3М в различных частях модельного кристалла были измерены спектры модального усиления-поглощения в зависимости от плотности токов накачки по методике из [8]. Для этого были изготовлены образцы $600 \times 600$ мкм с контактом, разделенным электрически изолирующими мезаканавками на продольные сегменты шириной 200 мкм. Одна из боковых граней, параллельная сегментам 200 мкм, служила выходной гранью, откуда осуществлялась регистрация излучения, а остальные три грани изготавливались шероховатыми. Образец монтировали на теплоотвод подложкой вниз, выходной гранью наружу и накачивали импульсами тока длительностью 3-5 мкс. При накачке ближайшего к выходной грани сегмента регистрировался опорный спектр электролюминесценции при плотности тока $150 \mathrm{~A} / \mathrm{cm}^{2}$. Далее током с той же плотностью накачивали следующий от выходной грани сегмент, а ближайший к выходной грани сигнальный сегмент накачивали варьируе-

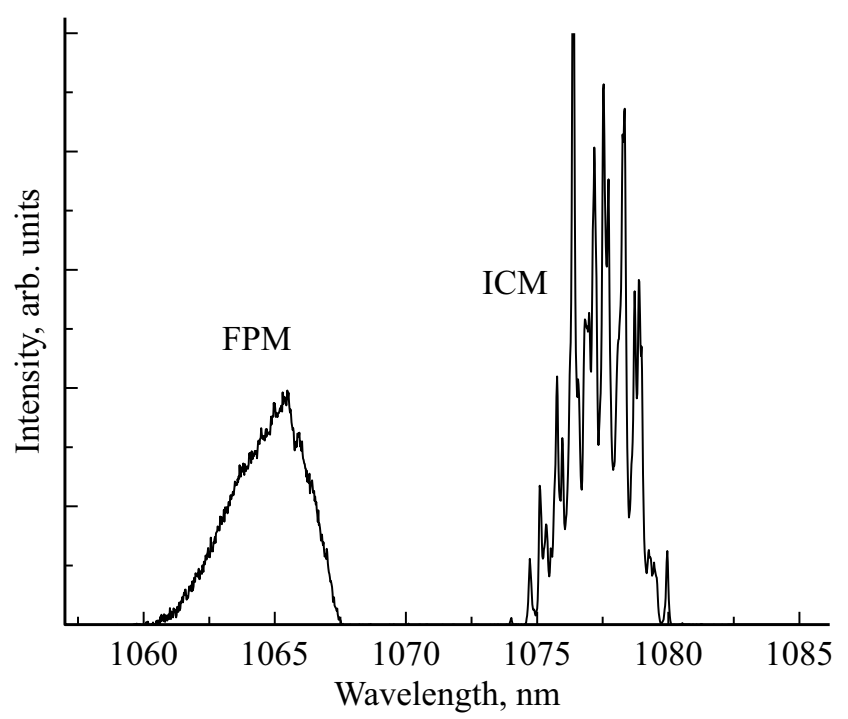

Рис. 2. Типичные спектры генерации замкнутой моды (ICM, кристалл $500 \times 500$ мкм) и моды резонатора Фабри-Перо (FPM, резонатор 100 мкм $\times 2.5$ мм) при плотности тока непрерывной накачки 1 кА/см² каждый. 
мыми плотностями тока. Таким образом получался сигнальный спектр излучения, прошедшего через сегмент с варьируемыми условиями накачки. По соотношению из [8] рассчитывали спектральные зависимости модального усиления-поглощения для различных плотностей токов накачки сигнального сегмента. Измеренные спектры представлены на рис. 3. В работе [4] для описания усиления ЗМ было предложено использовать зависимость для ФПМ за вычетом константы, описывающей расстройку по величине усиления. В случае значительной вариации геометрических параметров модельного кристалла-излучателя $3 \mathrm{M}$ (рис. 1) величина пороговой плотности тока также будет изменяться в широком диапазоне, и расстройки усиления в виде константы недостаточно для адекватного описания пороговых условий работы ЗМ. С учетом характерной величины смещения линии ЗМ в длинноволновую сторону (рис. 2) и вида спектральных зависимостей модального усиления от концентрации (рис. 3) зависимость модального усиления от концентрации для 3М можно представить как

$$
\Gamma_{\mathrm{QW}} g_{\mathrm{mat}}^{\mathrm{CM}}=K \Gamma_{\mathrm{QW}} g_{\text {mat }}^{\mathrm{FP}},
$$

где $g_{\mathrm{mat}}^{\mathrm{FP}}-$ зависимость материального усиления от концентрации носителей для $Ф П \mathrm{M}, \Gamma_{\mathrm{QW}}-$ поперечный фактор оптического ограничения активной области (в рамках данной работы предполагается, что поперечные профили ФПМ и ЗМ совпадают), $K$ - коэффициент, отражающий тот факт, что линия генерации $3 \mathrm{M}$ лежит в стороне от максимума спектра усиления активной области. Величина коэффициента $K$ выбирается для конкретного лазерного кристалла на основе спектров модального усиления-поглощения (рис. 3), характерное значение составляет 0.7-0.9. Для описания $g_{\mathrm{mat}}^{\mathrm{FP}}$ в практических расчетах можно использовать хорошо себя зарекомендовавшую логарифмическую зависимость материального усиления от концентрации для ФПМ [1], которую легко построить на основе данных стандартной характеризации лазерной гетероструктуры на серии образцов лазеров с ФПМ [9].

Потери оптической мощности ЗМ внутри лазерного кристалла можно разделить на потери, связанные с активной областью и ее накачкой в виде межзонного поглощения и потерь на свободных носителях заряда, потери на вывод излучения и прочие потери: на дефектах в волноводе и эпитаксиальных интерфейсах, рэлеевское рассеяние и вследствие эффектов, проявляющихся при высоких уровнях накачки [10]. С учетом этого выражение для суммарных потерь ЗМ можно записать в виде

$$
\alpha_{\mathrm{CM}}^{\Sigma}=\alpha_{i} \Gamma_{p}+\alpha_{\mathrm{CM}}^{\mathrm{BGA}}\left(1-\Gamma_{p}\right)+\alpha_{i 0}+\alpha_{\mathrm{CM}}^{\mathrm{out}},
$$

где $\alpha_{i}$ - оптические потери на свободных носителях заряда в активной области и волноводе сегмента накачки, соответствующие внутренним оптическим потерям для мощных ФПМ-лазеров, $\Gamma_{p}-$ планарный фактор оптического ограничения ЗМ в области усиления лазерного кристалла, $\alpha_{\mathrm{CM}}^{\mathrm{BGA}}-$ потери на межзонное
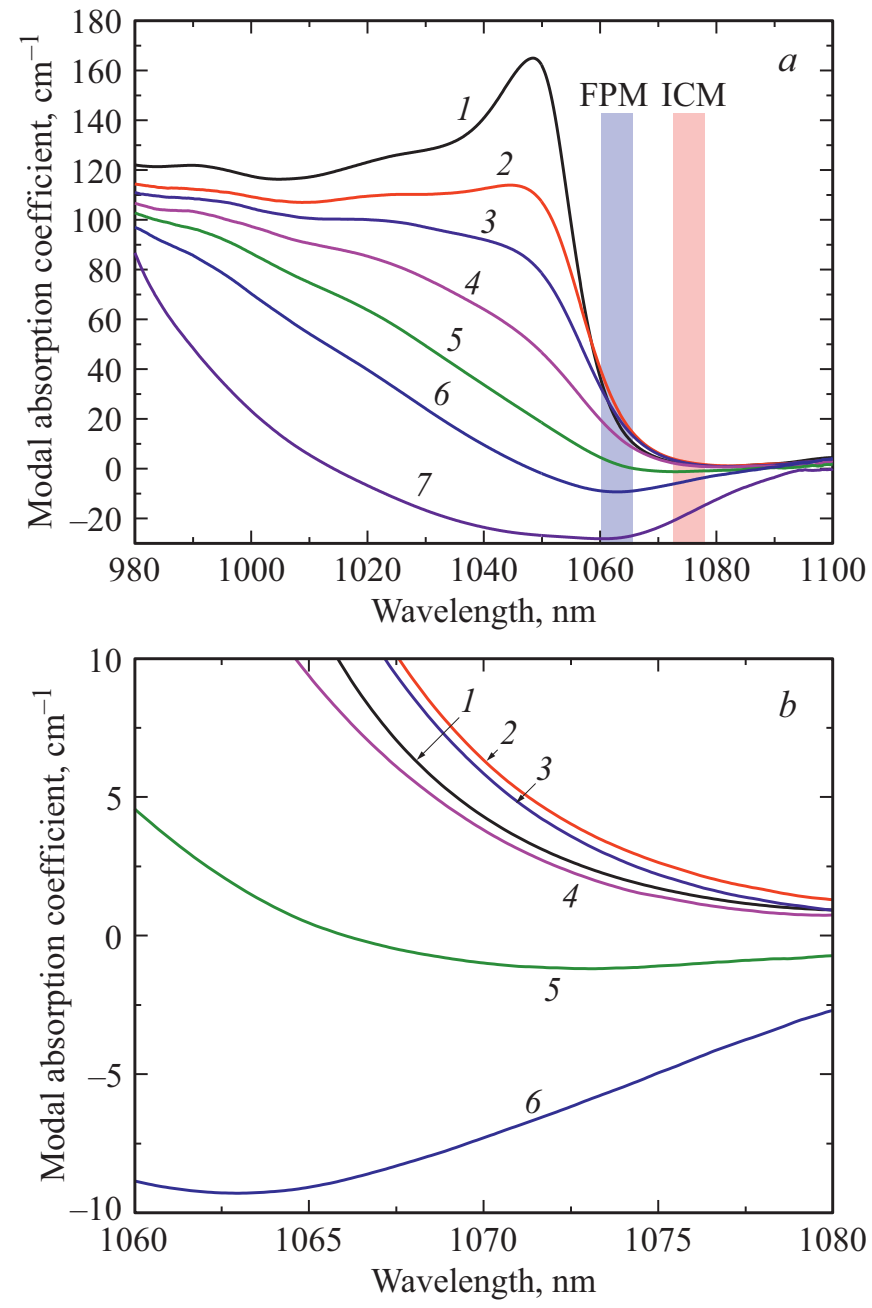

Рис. 3. $a-$ общий вид спектров поглощения-усиления в зависимости от плотности накачки активной области. $b-$ поглощение-усиление в области длин волн генерации ЗМ. Плотности тока накачки $J, \mathrm{~A} / \mathrm{cm}^{2}: 1-0,2-5,3-20$, $4-50,5-100,6-200,7-500$. Схематично указаны типичные спектральные области генерации мод ФПМ и ЗМ.

поглощение в пассивной части кристалла, $\alpha_{i 0}-$ потери, связанные с фоновой концентрацией легирующих примесей, эпитаксиальными несовершенствами и рассеянием в волноводных слоях гетероструктуры, $\alpha_{\mathrm{CM}}^{\text {out }}-$ потери на вывод излучения 3М из кристалла. Характерные величины слагаемых выражения (2) составляют: $\alpha_{i}=0.7 \mathrm{~cm}^{-1}, \alpha_{\mathrm{CM}}^{\mathrm{BGA}}=0.5-3 \mathrm{~cm}^{-1}$ (рис. $3-$ для области длин волн, соответствующей типичной линии генерации $3 \mathrm{M}), \alpha_{i 0}<0.1 \mathrm{~cm}^{-1}, \alpha_{\mathrm{CM}}^{\text {out }}$ будут определяться конструкцией области вывода излучения $3 \mathrm{M}$ из кристалла. Рассмотрим подробнее характер поведения $\alpha_{\mathrm{CM}}^{\mathrm{BGA}}-$ потерь 3М на межзонное поглощение в активной области пассивной части кристалла. С увеличением уровня накачки активного сегмента кристалла (область 2 на рис. 1) увеличивается засветка пассивной части (область 3 на рис. 1) спонтанным излучением и вследствие межзонного поглощения происходит возрастание 


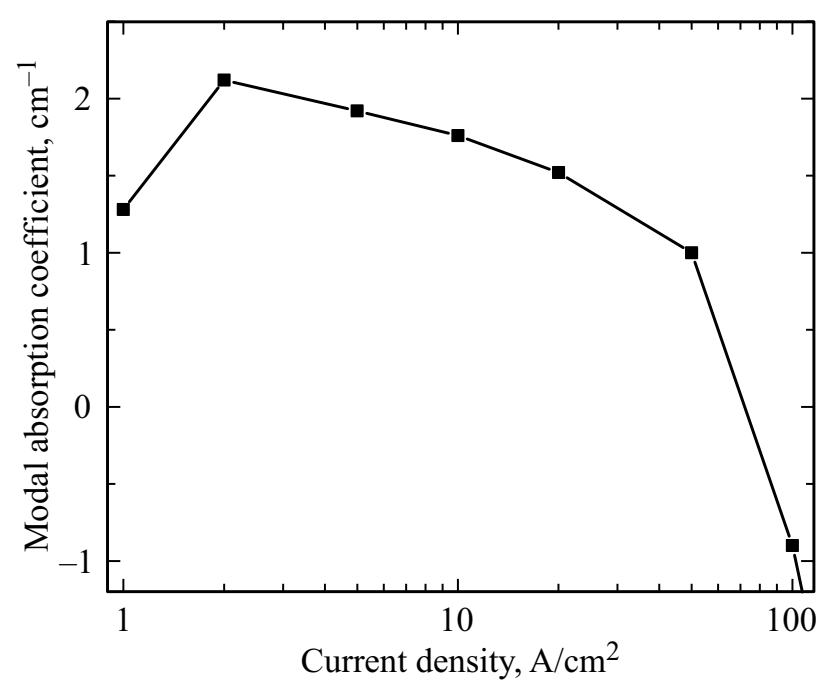

Рис. 4. Зависимость коэффициента поглощения от плотности тока накачки на длине волны 3М (1077 нм).

концентрации носителей заряда в активной области. Это приводит к разрушению краевого пика поглощения и модификации формы края спектра поглощения в области длин волн генерации 3М (рис. 3). Из рис. 3, $b$ видно, что первоначальный рост концентрации носителей в квантовой яме приводит к возрастанию поглощения в области длин волн генерации ЗМ. Это можно связать с нетепловым уменьшением ширины запрещенной зоны вследствие эффектов многочастичного взаимодействия при росте концентрации носителей в активной области [11-14]. С дальнейшим увеличением засветки и концентрации носителей происходит уменьшение потерь на межзонное поглощение. Итоговая зависимость, представленная на рис. 4, показывает, что минимальные потери $3 \mathrm{M} \alpha_{\mathrm{CM}}^{\mathrm{BGA}}$ в пассивной части кристалла можно получить: 1) в случае минимальной концентрации носителей заряда в активной области, при которой не происходит сдвига края поглощения в длинноволновую область; 2) в случае повышения концентрации до значительных уровней вследствие засветки спонтанным излучением из области усиления. Первый случай возможно обеспечить за счет изготовления омических контактов в пассивной части и обеспечения короткого замыкания $p-n$-перехода для сброса концентрации фотогенерированных в активной области носителей заряда [5], второй случай увеличением фактора $\Gamma_{p}$ для эффективной засветки пассивной части кристалла. Выражение для пороговых условий ЗМ в кристалле можно записать в виде

$$
K \Gamma_{\mathrm{QW}} g_{\mathrm{mat}}^{\mathrm{FP}} \Gamma_{p}=\alpha_{i} \Gamma_{p}+\alpha_{\mathrm{CM}}^{\mathrm{BGA}}\left(1-\Gamma_{p}\right)+\alpha_{i 0}+\alpha_{\mathrm{CM}}^{\mathrm{out}} .
$$

Из данного выражения видно, что при низких значениях фактора планарного ограничения области усиления 3М $\Gamma_{p}$ будет происходить значительное повышение порогового значения материального усиления и пороговой плотности тока накачки.

\section{4. Эффективность генерации замкнутых мод и конструкции лазерного кристалла}

С учетом описания потерь 3М (2) выражение для дифференциальной эффективности [1] можно записать в виде

$$
\eta_{d}=\eta_{i} \frac{\alpha_{\mathrm{CM}}^{\text {out }}}{\alpha_{i} \Gamma_{p}+\alpha_{\mathrm{CM}}^{\mathrm{BGA}}\left(1-\Gamma_{p}\right)+\alpha_{i 0}+\alpha_{\mathrm{CM}}^{\text {out }}},
$$

где $\eta_{i}$ - внутренний квантовый выход лазерной гетероструктуры, учитывающий квантовую эффективность активной области и эффективность токовой инжекции в нее. Из выражения (4) видно, что для получения высокой дифференциальной эффективности излучателя необходимо снижать суммарные оптические потери 3М. Рассмотрим подробнее слагаемые в (2) и их влияние на эффективность лазерной генерации ЗМ. Поскольку предлагаемый излучатель на основе $3 \mathrm{M}$ предполагает возможность получения достаточно узких линий лазерной генерации, то лазерный кристалл-резонатор должен обладать достаточно высокой добротностью, что означает работу в условиях низких потерь на вывод излучения из кристалла $\alpha_{\mathrm{CM}}^{\text {out }}$. В зависимости от величины планарного фактора ограничения 3М $\Gamma_{p}$ доминирующим механизмом потерь ЗМ будут либо внутренние оптические потери, связанные с накачкой активной области сегмента усиления $\alpha_{i}$, либо потери на межзонное поглощение $\alpha_{\mathrm{CM}}^{\mathrm{BGA}}$ в пассивном сегменте. Снижением фактора $\Gamma_{p}$ можно уменьшить влияние слагаемого $\alpha_{i} \Gamma_{p}$. Однако чрезмерное снижение фактора планарного ограничения $\Gamma_{p}$ будет приводить к значительному росту пороговой плотности тока и в свою очередь увеличивать $\alpha_{i}$. Это также будет приводить к насыщению усиления активной области и снижению рабочей величины $d g_{\text {mat }} / d n$, что снизит модуляционные характеристики прибора [1]. Таким образом, значительное уменьшение $\Gamma_{p}$ и $\alpha_{i} \Gamma_{p}$ невозможно без одновременного уменьшения потерь на межзонное поглощение в пассивных областях кристалла.

С учетом влияния фактора $\Gamma_{p}$ на пороговую плотность тока можно предложить два варианта конструкции кристалла-излучателя 3М. Первый вариант конструкции предполагает использование кристаллов с высокими значениями $\Gamma_{p}$, т. е. область усиления $3 \mathrm{M}$ распространяется на значительную часть ее траектории. В этом случае обеспечивается эффективная засветка пассивной части кристалла, низкая пороговая плотность тока в области усиления, но высокое значение слагаемого $\alpha_{i} \Gamma_{p}$ в суммарных потерях 3М и невысокие значения добротности резонатора ЗМ. На рис. 5 представлены расчетные зависимости дифференциальной эффективности данного типа конструкции для случая кристалла $1 \times 1$ мм на основе описанной выше гетероструктуры. Из расчетных зависимостей видно, что высокие дифференциальные эффективности достижимы лишь в случае высоких значений потерь на вывод излучения из резонатора, что 


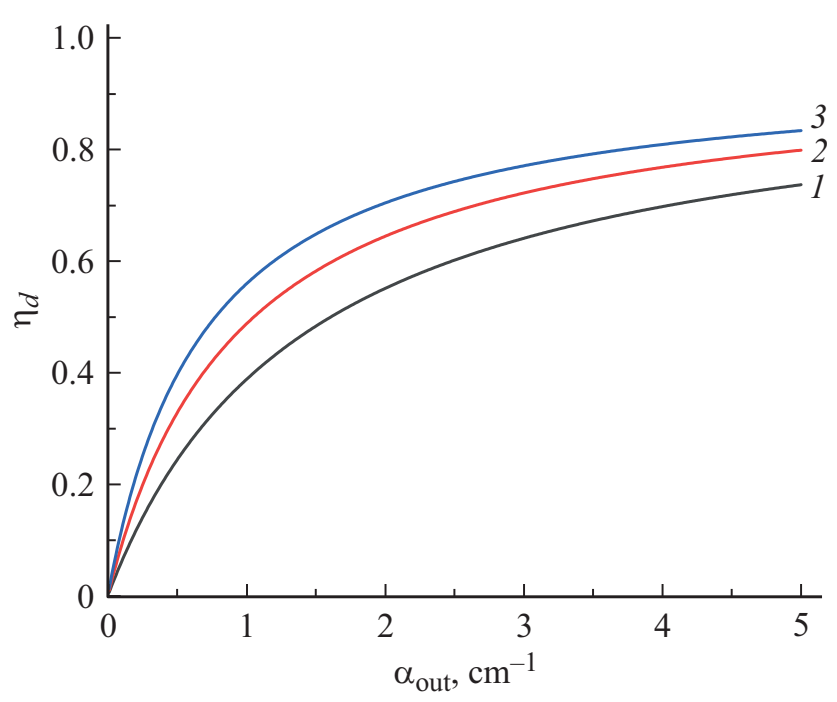

Рис. 5. Зависимости внешней дифференциальной эффективности от потерь на вывод излучения ЗМ для различных потерь ЗМ на межзонное поглощение в пассивной части кристалла, см ${ }^{-1}: 1-2,2-1,3-0.5$. Кристалл $1 \times 1$ мм при длине области усиления 1410 мкм (фактор ограничения 0.5 ); $\alpha_{i 0}=0.1 \mathrm{~cm}^{-1}, \alpha_{i}=0.7 \mathrm{~cm}^{-1}$.

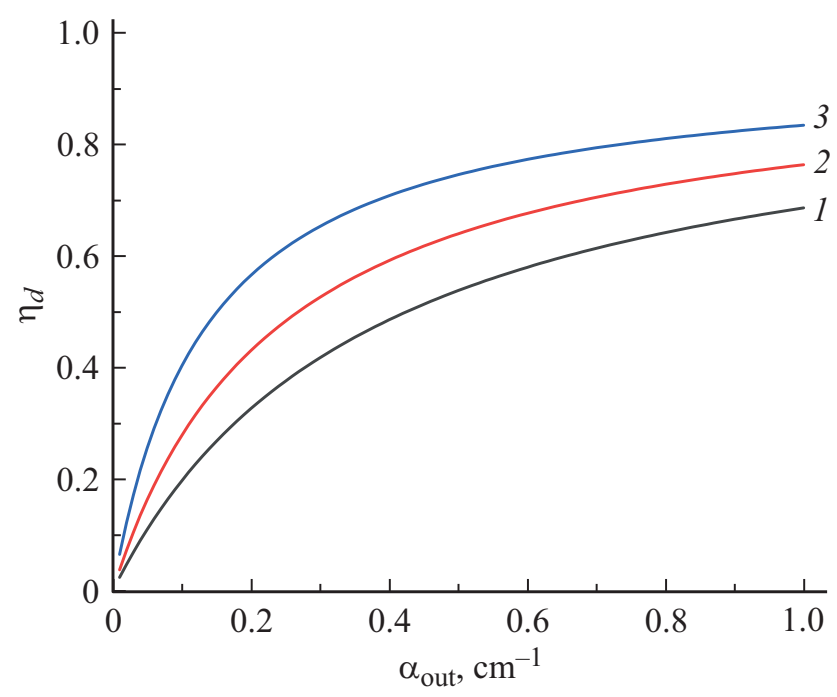

Рис. 6. Зависимости внешней дифференциальной эффективности от потерь на вывод излучения ЗМ в отсутствие потерь на межзонное поглощение в пассивной части кристалла при различных факторах ограничения активного сегмента $\Gamma_{p}: 1-$ $0.4,2-0.2,3-0.05$ (соответствуют длинам активного сегмента 1120,560 и 140 мкм при размере кристалла $1 \times 1$ мм); $\alpha_{i 0}=0.1 \mathrm{~cm}^{-1}, \alpha_{i}=0.7 \mathrm{~cm}^{-1}$.

также будет снижать добротность и не позволит сузить линии лазерной генерации. Альтернативным вариантом конструкции для снижения потерь ЗМ будет использование в пассивной части кристалла волноведущих слоев без активной области. Это возможно при применении двухстадийной эпитаксии. В этом случае выражения для пороговых условий и дифференциальной эффективности примут вид

$$
\begin{gathered}
K \Gamma_{\mathrm{QW}} g_{\mathrm{mat}}^{\mathrm{FP}} \Gamma_{p}=\alpha_{i} \Gamma_{p}+\alpha_{i 0}+\alpha_{\mathrm{CM}}^{\mathrm{out}}, \\
\eta_{d}=\eta_{i} \frac{\alpha_{\mathrm{CM}}^{\mathrm{out}}}{\alpha_{i} \Gamma_{p}+\alpha_{i 0}+\alpha_{\mathrm{CM}}^{\text {out }}} .
\end{gathered}
$$

Расчетные зависимости дифференциальной эффективности (рис. 6) в этом случае показывают, что в отсутствие межзонного поглощения в пассивной части кристалла возможно достижение высоких значений внешней дифференциальной эффективности при низких потерях $3 \mathrm{M}$ на вывод излучения. В этом случае возможно увеличение общей добротности резонатора и сужение линий лазерной генерации $3 \mathrm{M}$ с сохранением высокой эффективности излучателя.

\section{5. Заключение}

Проведен анализ пороговых условий и эффективности генерации замкнутой моды в прямоугольном кристалле большого размера $(1 \times 1$ мм $)$ на основе гетероструктуры для мощных полосковых лазеров Фабри-Перо с квантово-размерной активной областью. На основании экспериментальных данных показан нелинейный характер зависимости потерь замкнутой моды на межзонное поглощение в непрокачиваемой части лазерного кристалла. Рассмотрены условия достижения высокой внешней эффективности лазерной генерации замкнутой моды. Показано, что снижение потерь замкнутой моды на межзонное поглощение в непрокачиваемой части лазерного кристалла является определяющим фактором для достижения высокой эффективности генерации ЗМ. Рассмотрены варианты конструкции кристалла-излучателя как с активной областью в непрокачиваемой части лазерного кристалла, так и без нее, позволяющие достичь значений дифференциальной эффективности, характерных для лазеров полосковой конструкции.

\section{Финансирование работы}

В части экспериментальных исследований работа выполнена при финансовой поддержке Российского фонда фундаментальных исследований (проект 18-02-00835А). В части разработки технологии постростовых операций экспериментальных образцов исследование выполнено за счет Государственного задания ФТИ им. А.Ф. Иоффе.

\section{Конфликт интересов}

Авторы заявляют об отсутствии конфликта интересов.

\section{Список литературы}

[1] L.A. Coldren, S.W. Corzine, M.L. Mashanovitch. Diode lasers and photonic integrated circuits (N. Y., John Wiley \& Sons, Inc., 2012). 
[2] С.О. Слипченко, А.А. Подоскин, Д.А. Винокуров, А.Л. Станкевич, А.Ю. Лешко, Н.А. Пихтин, В.В. Забродский, И.С. Тарасов. ФТП, 45 (10), 1431 (2011).

[3] А.А. Подоскин, Д.Н. Романович, И.С. Шашкин, П.С. Гаврина, 3.Н. Соколова, С.О. Слипченко, Н.А. Пихтин. ФТП, $\mathbf{5 3}(6), 839$ (2019).

[4] С.О. Слипченко, А.А. Подоскин, Н.А. Пихтин, 3.Н. Соколова, А.Ю. Лешко, И.С. Тарасов. ФТП, 45 (5), 672 (2011).

[5] P. Blood, A.I. Kucharska, J.P. Jacobs, K. Griffiths. J. Appl. Phys., 70 (3), 1144 (1991).

[6] J. Stohs, D.J. Bossert, D.J. Gallant, S.R.J. Brueck. IEEE J. Quant. Electron., 37 (11) 1449 (2001).

[7] J. Hader, J.V. Moloney, S.W. Koch. IEEE J. Quant. Electron., 35 (12), 1878 (1999).

[8] С.О. Слипченко, А.А. Подоскин, Н.А. Пихтин, А.Л. Станкевич, Н.А. Рудова, А.Ю. Лешко, И.С. Тарасов. ФТП, $45(5), 682$ (2011).

[9] А.А. Подоскин, Д.Н. Романович, И.С. Шашкин, П.С. Гаврина, 3.Н. Соколова, С.О. Слипченко, Н.А. Пихтин. ФТП, 54 (5), 484 (2020).

[10] J. Piprek. Opt. Quant. Electron., 51 (2), 60 (2019).

[11] D.S. Chemla, D.A.B. Miller. J. Opt. Soc. Am. B, 2 (7), 1155 (1985).

[12] J.P. Reithmaier, R. Hoger, H. Riechert. Phys. Rev. B, 43 (6), 4933 (1991).

[13] G. Livescu, D.A.B. Miller, D.S. Chemla, M. Ramaswamy, T.Y. Chang, N. Sauer, A.C. Gossard, J.H. English. IEEE J. Quant. Electron., 24 (8), 1677 (1988).

[14] S.L. Chuang, N. Peyghambarian, S. Koch. Physics of optoelectronic devices (N.Y., Wiley, 1995).

Редактор Л.В. Шаронова

\title{
Analysis of threshold conditions and lasing efficiency of internally circulating mode in large rectangular cavities based on AIGaAs/InGaAs/GaAs laser heterostructures
}

\author{
A.A. Podoskin, D.N. Romanovich, I.S. Shashkin, \\ P.S. Gavrina, Z.N. Sokolova, S.O. Slipchenko, \\ N.A. Pikhtin \\ loffe Institute, \\ 194021 St. Petersburg, Russia
}

Abstract Threshold conditions and efficiency analysis of semiconductor laser emitters with a large rectangular resonator $(1 \times 1 \mathrm{~mm})$ based on $\mathrm{AlGaAs} / \mathrm{GaAs} / \mathrm{InGaAs}$ heterostructures for high-power strip lasers operating on high-Q internally circulating structures is presented. Two designs of emitters with different laser radiation propagation region characteristics are proposed, and possibility of differential efficiency characteristic as high as highpower broad lasers $(70 \%$ and more $)$ is demonstrated. 